\title{
Amino-Nogo Inhibits Optic Nerve Regeneration and Functional Recovery via the Integrin $\alpha v$ Signaling Pathway in Rats
}

\author{
Yan Huo ${ }^{\mathrm{a}, \mathrm{b}}$ Xiao-Lei Yin ${ }^{\mathrm{a}, \mathrm{c}}$ Shu-Xing Jia Huan Zou ${ }^{\mathrm{d}}$ Min Lang ${ }^{\mathrm{a}}$ Zheng Zhenga,e \\ Xiao-Feng Cai ${ }^{a}$ Wei Liu ${ }^{a}$ Chun-Lin Chen ${ }^{a}$ Yuan-Guo Zhou ${ }^{f}$ Rong-Di Yuan ${ }^{d}$ \\ Jian Ye
}

aDepartment of Ophthalmology, Institute of Surgery Research, Daping Hospital, Third Military Medical University, Chongqing, bepartment of Ophthalmology, 254 Hospital of PLA, Tianjin, 'Department of Ophthalmology, 305 Hospital of PLA, Beijing, dDepartment of Ophthalmology, Xinqiao Hospital, Third Military Medical University, Chongqing, eDepartment of Ophthalmology, The Second Affiliated Hospital of Chongqing Medical University, Chongqing, 'Department of Molecular Biology Center, State Key Laboratory of Trauma, Burn and Combined Injury, Institute of Surgery Research, Daping Hospital, Third Military Medical University, Chongqing, China

\section{Key Words}

Amino-Nogo $•$ Axonal regeneration $\bullet$ Optic nerve $\bullet$ Integrin $\bullet$ Signaling pathway

\begin{abstract}
Background: Nogo-A, a major myelin-associated inhibitor, can inhibit injured optic nerve regeneration. However, whether Amino-Nogo is the most important functional domain of Nogo-A remains unknown. This study aimed to identify the role of Amino-Nogo following optic nerve injury, and the mechanism of the Amino-Nogo-integrin av signaling pathway in vivo. Methods: Sprague-Dawley rats with optic nerve crush injury were injected with Nogo-A siRNA (Nogo-A-siRNA), the Nogo-66 functional domain antagonist peptide of Nogo-A (Nep1-40) or a recombinant rat Amino-Nogo-A protein $(\Delta 20)$ into the vitreous cavity to knock down Nogo-A, inhibit Nogo-66 or activate the Amino-Nogo, resparately. Retinal ganglion cell (RGC) density, axon regeneration and the pattern of NPN of visual electrophysiology (flash visual evoked potentials [F-VEP]) at different times post-injury were investigated. Results: Our study revealed a lower RGC survival rate; shorter axonal outgrowth; longer N1, P1 and N2 waves latencies; and lower N1-P1 and P1-N2 amplitudes in the $\Delta 20$ group, and $\Delta 20$ treatment significantly attenuated integrin $\alpha v$ expression and phosphorylated focal adhesion kinase ( $p$-FAK) levels. In the Nep1-40 and Nogo-A siRNA groups, there were higher RGC survival rates, longer axonal outgrowth, shorter N1 and P1 wave latencies, and higher N1-P1 and P1-N2amplitudes. Nogo-A siRNA treatment significantly increased integrin av expression and p-FAK levels. Nepl-40 treatment did not alter integrin $\alpha v$ expression. In addition, there was no significant change in integrin $\alpha 5$ in any group. Conclusion: These results suggest that the integrin signaling pathway is regulated by Amino-Nogo, which inhibits optic nerve regeneration and functional recovery, and that the integrin subunit involved might be integrin $\alpha v$ but not integrin $\alpha 5$.
\end{abstract}

Copyright $\odot 2015$ S. Karger AG, Basel

Jian Ye

and Rong-Di Yuan

\section{KARGER 125}

Department of Ophthalmology, Institute of Surgery Research, Daping Hospital, Third Military Medical University, Chongqing, 400042 (China) and Department of Ophthalmology, Xinqiao Hospital, Third Military Medical University, Chongqing, 400037 (China)

E-Mail dpyyyk@163.com, E-Mail yuanrongdi@126.com 


\section{Introduction}

Eyes detect light and convert it into electro-chemical impulses in the retina, which is highly organized neural tissue and has been studied widely in neuroscience [1]. Retinal ganglion cell (RGC) axons form the optic nerve, which is a part of the central nervous system (CNS). Following injury, the optic nerve is unable to regenerate, which can result in visual loss. Studies show that failure of axonal regeneration in the adult mammalian CNS is partly due to the presence of endogenous inhibitors of regeneration, which include Nogo-A, myelinassociated glycoprotein (MAG), and oligodendrocyte myelin glycoprotein (OMgp).

Nogo-A [2-4] has received much attention as an endogenous inhibitor, and its function depends on its two inhibitory domains: the amino-terminu (172 amino acids) and the Nogo66 loop (located between two hydrophobic domains) [2, 4-5]. Nogo-66 combined with its receptor ( $\mathrm{NgR}$ ) [5] plays a dual role in axonal regeneration, where it has been reported to regulate axonal growth, guidance and CNS neurogenesis and plasticity, with a sole inhibitory effect of Amino-Nogo on axonal regeneration having also been reported [6-11]. In vitro studies have shown that Amino-Nogo is an important inhibitory domain of RGC axonal outgrowth and the mechanism may act via the integrin $\alpha \mathrm{v}$ signaling pathway [12]. Furthermore, AminoNogo-integrin signaling pathway-related proteins (Nogo-A [13], integrin $\alpha v$, integrin $\alpha 5$ and focal adhesion kinase (FAK)) are expressed in the visual system. However, whether AminoNogo can promote optic nerve regeneration and the mechanism of Amino-Nogo-integrin signaling pathway action in vivo, remain unknown. This study aimed to investigate the effects of Amino-Nogo in optic nerve injury and its possible signaling pathway, and thereby to provide a basis for promoting optic nerve regeneration and functional recovery. Optic nerve-injured Sprague-Dawley (SD) rats were transfected with Nogo-A small interfering RNA (siRNA) and injected with a Nogo-66 antagonist peptide (Nep1-40) or a recombinant AminoNogo Fc chimera $(\Delta 20)$ into the vitreous cavity. RGC survival rate, axonal regeneration and visual functional recovery were observed. Our results demonstrate that the Amino-Nogo domain decreased RGC survival, inhibited axonal regeneration and did harm to the recovery of neurological functions. Furthermore, all of these actions were mediated by the integrin $\alpha \mathrm{v}$ signaling pathway.

\section{Materials and Methods}

Materials and animals

Nogo-66 (1-40) antagonist peptide (Nep1-40) was purchased from Sigma Aldrich (MO, USA). Recombinant rat NogoA Fc chimera $(\triangle 20)$ was purchased from R\&D Systems (MN, USA), and a RhoA activation assay kit was purchased from Cytoskeleton (CO, USA). pAKD.CMV.bGlobin.eGFP.H1.shRNA was purchased from Neuron Biotech (SH, China). Antibodies targeting Nogo-A, integrin $\alpha \mathrm{v}$, integrin $\alpha 5$, FAK, and p-FAK were purchased from Santa Cruz (CA, USA). Enhanced chemiluminescence (ECL) substrate was purchased from Pierce (CO, USA). FluoroGold (FG) was purchased from Fluorochrome (CO, USA).

Adult SD rats of either sex weighing 180-220 g were provided by the Animal Experimental Center (Institute of Surgery Research, Daping Hospital, Third Military Medical University, China). The Animal Research Committee of the Third Military Medical University approved the study protocol.

Optic nerve crush injury

Surgical procedures were based on a previously described method [14,15]. Briefly, adult SD rats were anesthetized with pentobarbital sodium (30 mg/kg, i.p.). Optic nerve crush injury was induced under a binocular surgical micro-scope. The right optic nerve of each animal was subjected to a crush injury with a vascular clip 1-2 $\mathrm{mm}$ posterior to the globe for $10 \mathrm{~s}$ [16]. Sham-operated animals underwent surgery in which the optic nerve was exposed but not crushed.

Virus transduction and intra-ocular injections

Small interfering RNA (siRNA) duplexes targeted the Nogo-A sequence: 5'-GATCCAGGC TATCCAGAAA-3'. The negative control (NC) sequence was: 5'-TTCTCCGAACGTGTCACGT-3'. An H1 RNA polymerase III 
promoter drove the expression of Nogo-A. The Nogo-A shRNA was packaged with pAKD.CMV.bGlobin.eGFP. H1.shRNA. Large-scale recombinant adeno-associated virus 2/8 (rAAV2/8) production and purification were performed as previously described [17].

Intravitreal injections were made through the sclera just behind the cornea with a 30 -gauge needle micro-pipette. Approximately $10^{12} \mathrm{AAV}$ particles in $10 \mu \mathrm{l}$ phosphate-buffered saline (PBS) were injected into the vitreous cavity 3 weeks before optic nerve surgery. Nep1-40 (500 nM) and $\Delta 20(400 \mathrm{nM})$ were injected after the optic nerve crush. PBS $(10 \mu \mathrm{l})$ was injected as the control group.

\section{Retrograde labeling and counting of RGCS}

One week before death, the rats were anesthetized with pentobarbital sodium (30 mg/kg, i.p.) placed in a stereotactic apparatus and 3\% FG was injected into both superior colliculi. To count RGCs, we divided the retina into four parts (four quadrants). Three observation areas were selected at a distance of $1 / 6,1 / 2$, and $5 / 6$ of the retinal radius from the optic nerve disc at each radius quadrant. RGC densities were measured in 12 visual fields and counted according to a double-blind method by three different investigators. The RGC survival rate was presented as the ratio of the surviving RGC density after injury versus the sham-operated RGC density at the same time point.

\section{Quantification of axonal growth}

Adult SD rats were anesthetized with pentobarbital sodium (30 mg/kg, i.p.). The optic nerve was cut into $10 \mu \mathrm{m}$ slices and visualized by immunofluorescent staining of growth associated protein-43 (GAP-43). The number of regenerating axons at designated distances from the end of the crush sites was evaluated per section as previously reported [18]. The number of regenerating axons per nerve was then averaged over all sections of one nerve. Axon counts were converted into axon crossings (axons $/ \mathrm{mm}$ ). . ad, defined as the total number of axons extending the distance of an optic nerve with a radius of $r$, was estimated by summing over all sections of thickness $t$ as follows: $\Sigma$ ad $=\pi r^{2} \times($ average axons $/ \mathrm{mm}$ width) $/ \mathrm{t}$.

\section{F-VEP recording}

A VETS-3 vision electrophysiological diagnostic apparatus (Electric Appliance Factory, Chongqing, China) was used, in accordance with the International Society for the Clinical Electrophysiology of Vision (ISCEV) standard for electrophysiological studies. Following 15 minutes of dark adaptation, flash visual evoked potentials (F-VEP) were recorded using silver needle electrodes, which were implanted into the eminentia post occiput. A reference electrode was implanted into the midpoint of the binoculus and ground electrode was implanted into the tail of the rat. White flash stimuli were delivered at a frequency of $2 \mathrm{~Hz}$, $250 \mathrm{~ms}$ for analysis. The responses were amplified 10,000 times and band-pass-filtered from 1 to 1000 $\mathrm{Hz}$, and superposition was conducted 100 times. Stable wave forms were recorded three times in each eye and the contralateral eye was shaded with an eyeshade. The parameters observed were F-VEP latency (N1, P1, N2 wave response time, ms), N1-P1 amplitude (from N1 wave peak to P1 wave trough, mV) and P1$\mathrm{N} 2$ amplitude (from P1 wave trough to N2 wave peak, $\mathrm{mV}$ ). All of the parameter values were measured automatically by computer output, and the average of the three measurements was calculated.

\section{Western blotting}

Optic nerves were collected from rats at 14 days following injury. Protein samples were electrophoretically resolved using 10\% SDS-PAGE and transferred onto PVDF membranes. The membranes were blocked for 2 hours at room temperature in 5\% skim milk powder diluted with Tris-buffered saline contaning Tween-20. The membranes were incubated overnight at $4{ }^{\circ} \mathrm{C}$ with diluted anti-GAP-43, antiintegrin $\alpha v$, anti-integrin $\alpha 5$, anti-p-FAK, anti-FAK (1:500) or anti- $\beta$-actin (1:2000) antibodies in $5 \%$ milk. The membranes were incubated with horseradish peroxidase (HRP)-conjugated secondary antibodies for 1 hour at room temperature (rabbit anti-mouse HRP and goat anti-rabbit HRP, 1:5000). The signals were detected using ECL-Plus and exposed to film. The signal intensity of each band on the western blot was quantified using Labwork 4.6 and normalized to $\beta$-actin.

\section{Statistical analysis}

All of the results are reported as the mean \pm SD of at least six independent experiments. Comparisons of two groups were made using the unpaired t-test. Multiple-group statistical analyses were performed 

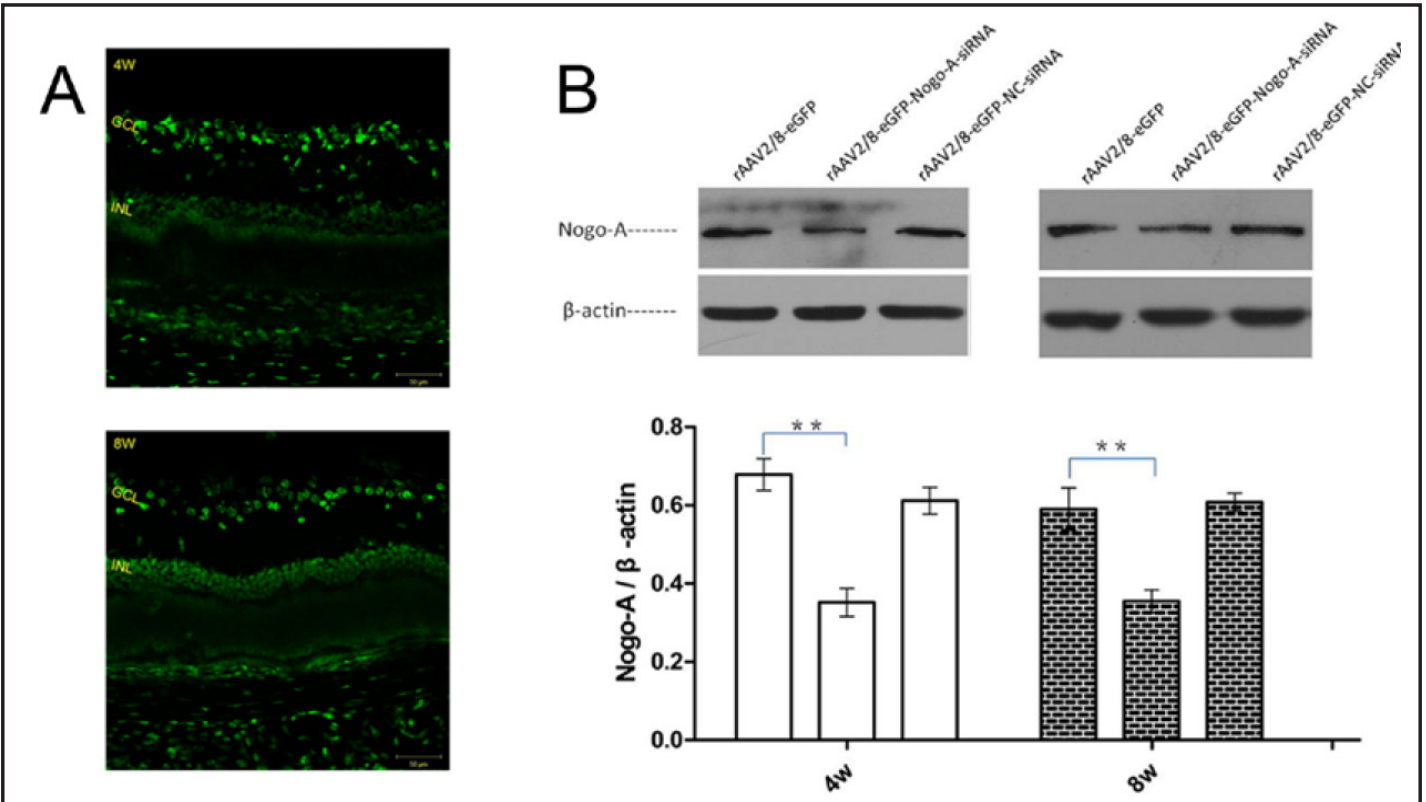

Fig. 1. Nogo-A expression in different rAAV2/8-Nogo-A-siRNA-transfected RGCs. The RGCs were transfected with rAAV2/8-eGFP, rAAV2/8-NC-siRNA or rAAV2/8-Nogo-A-siRNA for 4 and 8 weeks. A: Representative images showing rAAV2/8-eGFP expression in retinal sections. B: Representative western blotting and summary data showing Nogo-A protein expression at 4 (left panel) and 8 weeks (right panel) after rAAV injection. Error bars $=\mathrm{SD}, \mathrm{n}=6 .{ }^{* *}$ vs. $\mathrm{rAAV} 2 / 8-\mathrm{eGFP}, \mathrm{p}<0.01$.

using one-way analysis of variance (ANOVA), followed by least significant difference post hoc tests. All statistical analyses were performed using SPSS software (version 10.0 for Windows; SPSS Inc., Chicago, IL, USA). P values less than 0.05 were considered statistically significant.

\section{Results}

Efficient knockdown of Nogo-A with rAAV2/8-Nogo-A-siRNA in vivo

To monitor RGC transfection with $\mathrm{rAAV} 2 / 8$, we injected $\mathrm{rAAV} 2 / 8$ expressing the eGFP gene (rAAV2/8-eGFP) into the vitreous cavity. rAAV2/8-eGFP expression was detected in retinal sections (Fig. 1A), confirming that the rAAV transfection of RGCs was successful. To further confirm the efficacy of the RNAi, we examined Nogo-A protein levels by western blot analysis 4 and 8 weeks after rAAV injection. rAAV2/8-eGFP-Nogo-A-siRNA caused the greatest knockdown of Nogo-A when compared with the rAAV2/8-eGFP groups $(\mathrm{p}<0.01)$, whereas the rAAV2/8-eGFP-NC-siRNA and rAAV2/8-eGFP groups showed had no significant difference in Nogo-A level $(p>0.05)$ at the 4 and 8-week time points. This observation confirmed that the siRNA-mediated knockdown was a specific and significant response to Nogo-A, and that rAAV-2/8 was an effective transfection vehicle to deliver siRNA into RGCs (Fig. 1B).

\section{Amino-Nogo inhibits RGC survival in optic nerve-injured rats}

RGCs densities were detemined to analyze RGC survival. To assess RGC loss in PBS control, NC siRNA, Nogo-A siRNA, Nep1-40 (500 nM), and $\Delta 20$ (400 nM)-injected RGCs, we retrogradely labeled RGC neurons by injecting FG into the superior colliculus. The RGC survival rate of each group is presented in Fig. 2D. At day $7 \mathrm{~d}$ following optic nerve injury (Fig. $2 \mathrm{~A}$ ), the RGC survival rate in the Nogo-A siRNA-transfected group was significantly higher than in the PBS control $(p<0.05)$. The RGC survival rates were $62.76 \%$ in the Nep1-40 injected group and $51.28 \%$ in the PBS control group, but there was no significant difference 


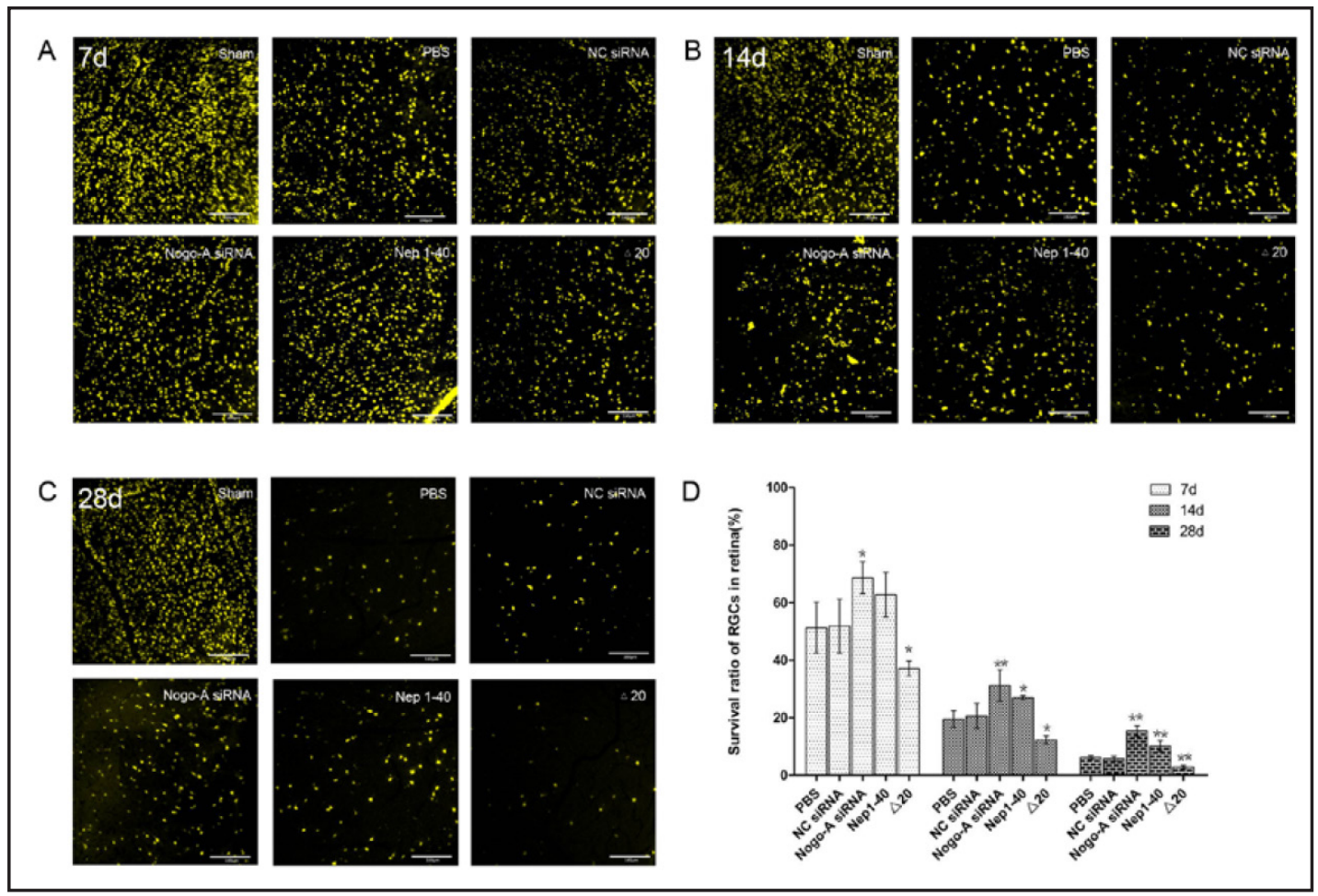

Fig. 2. Status of RGC survival in different groups 7, 14 and 28 days following optic nerve crush injury. A-C: Representative images of RGCs treated with sham control, PBS control, NC siRNA, Nogo-A siRNA, Nep1-40 (500 nM), or $\Delta 20(400 \mathrm{nM})$ at 7 (A), 14 (B) and 28 days (C) following optic nerve injury. D: Bar charts of quantitative analysis. Error bar $=\mathrm{SD}, \mathrm{n}=6{ }^{*}$ vs. PBS control, $\mathrm{p}<0.05,{ }^{* *}$ vs. PBS control, $\mathrm{p}<0.01$.

between them $(p>0.05)$. However, rats injected with $\Delta 20$ exhibited a significant reduction of RGC survival in comparison with the PBS control ( $p<0.05$ ). At 14 (Fig. 2B) and 28 days (Fig. 2C) following optic nerve injury, significantly higher RGC survival rates were observed in both the Nogo-A siRNA- and Nep1-40-treated groups compared to the PBS control ( $\mathrm{p}<$ $0.05)$, whereas a decreased RGC survival rate was observed in the $\Delta 20$-injected group. There was no difference between the NC siRNA- and PBS-treated groups at day 7, 14 or 28 (p > $0.05)$.

\section{Amino-Nogo inhibits axonal regeneration in optic nerve-injured rats}

GAP-43 was used to evaluate newly-outgrown axons from the soma [19] (Fig. 3A). At day 7 following the indicated treatments (Fig. 3B), the mean estimated numbers of outgrown axons (200 $\mu \mathrm{m}$ distal to lesion site) were significantly higher in the Nogo-A siRNA group (p $>0.01$ ) in comparison with the PBS group, whereas the shortest GAP-43 positive axons were seen in the $\Delta 20$ group $(\mathrm{p}<0.01$ ). In addition, axons growing $400 \mu \mathrm{m}$ distal to the lesion site were detected in the Nogo-A siRNA and Nep1-40 groups, and they were significantly longer than the PBS group ( $<<0.01)$. Furthermore, axons were significantly shorter in the $\triangle 20$ group than in the PBS group $(\mathrm{p}<0.01)$. At day 14 following the indicated treatments (Fig. 3C), the mean estimated numbers of outgrown axons (400 $\mu \mathrm{m}$ distal to lesion site) were significantly higher in the Nogo-A siRNA group $(\mathrm{p}<0.01)$ in comparison to the PBS group, whereas no GAP-43-positive axons were observed in the $\triangle 20$ group compared with the PBS group. At $800 \mu \mathrm{m}$ distal to the lesion site, GAP-43-positive axons were detected in the Nogo-A siRNA and Nep1-40 groups, and they were significantly longer than those in the PBS group $(\mathrm{p}<0.01)$. Axons were also detected in $\Delta 20$ group, and they were significantly shorter than those in the PBS group $(p<0.01)$. At day 28 following the indicated treatments (Fig. 3D), the mean estimated numbers of outgrown axons (400 and $800 \mu \mathrm{m}$ distal to lesion 


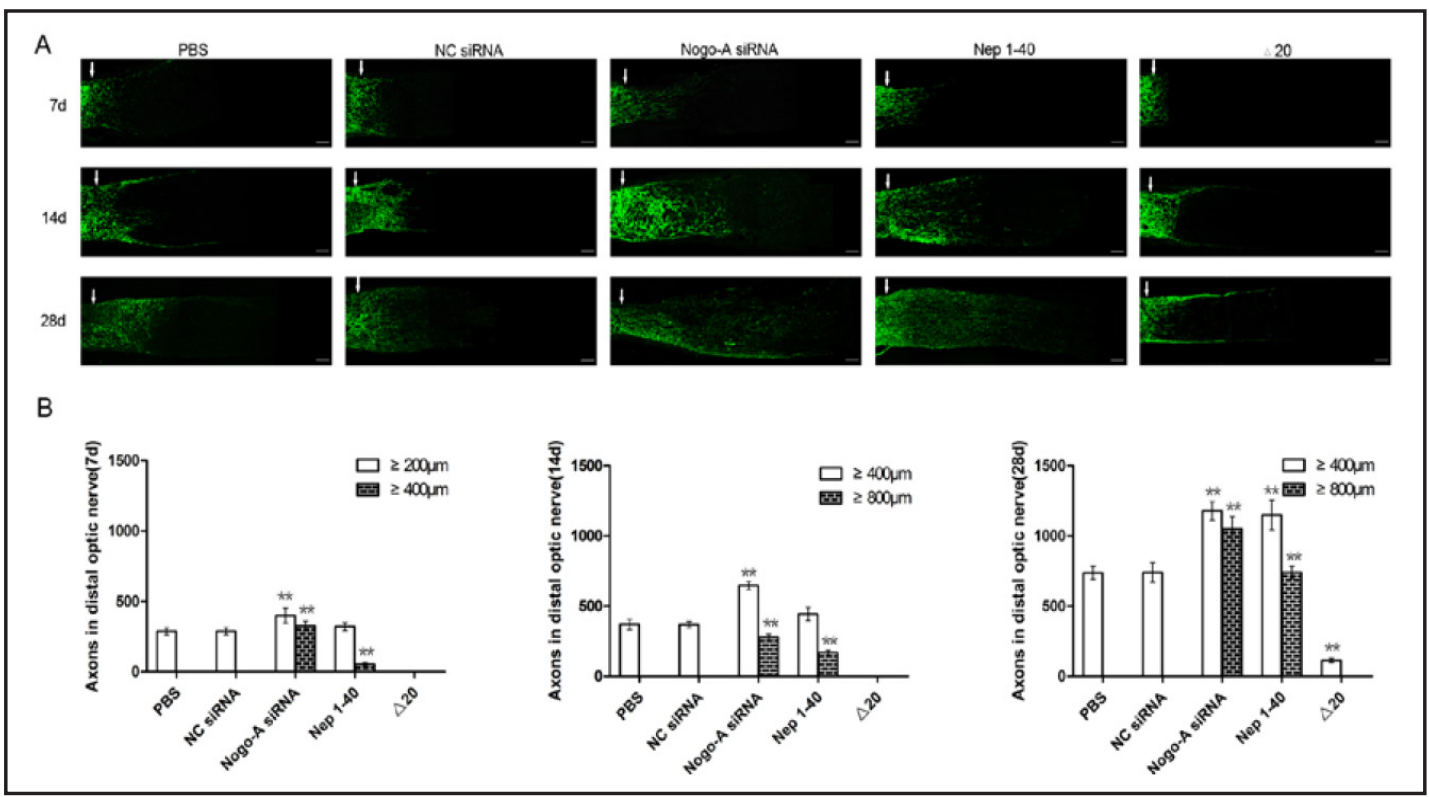

Fig. 3. Lengths of regenerated optic nerve axons in the indicated groups. A: Representative images showing longitudinal sections through optic nerve axons distal to the injury site immunostained for growth-associated protein 43 at 7, 14 and 28 days following nerve crush. B: Bar charts representing the estimated number of regenerated fibers at $7 \mathrm{~d}(\mathrm{~B}), 14 \mathrm{~d}$ (C) and 28d (D) following optic nerve injury. The arrow indicates the injury site; Scale bar $=100 \mu \mathrm{m}$; Error bar $=$ SD, $\mathrm{n}=6$. *vs. PBS control, $\mathrm{p}<0.05$; ** vs. PBS control, $\mathrm{p}<0.01$.

Fig. 4. Evaluation of the recovery of injured optic nerves by NPN wave pattern. A: Representative flash VEP tracings at 28 days following optic nerve crush injury in PBS, NC siRNA-, Nogo-A siRNA-, Nep1-40 (500 $n M)-$, and $\triangle 20 \quad(400$ nM)-treated groups. B: Bar charts showing F-VEP latency. C: Bar charts showing F-VEP amplitude. Error bars = SD, $\mathrm{n}=6$. *vs. PBS control, $\mathrm{p}<0.05$; ** vs. PBS control, $\mathrm{p}<0.01$.

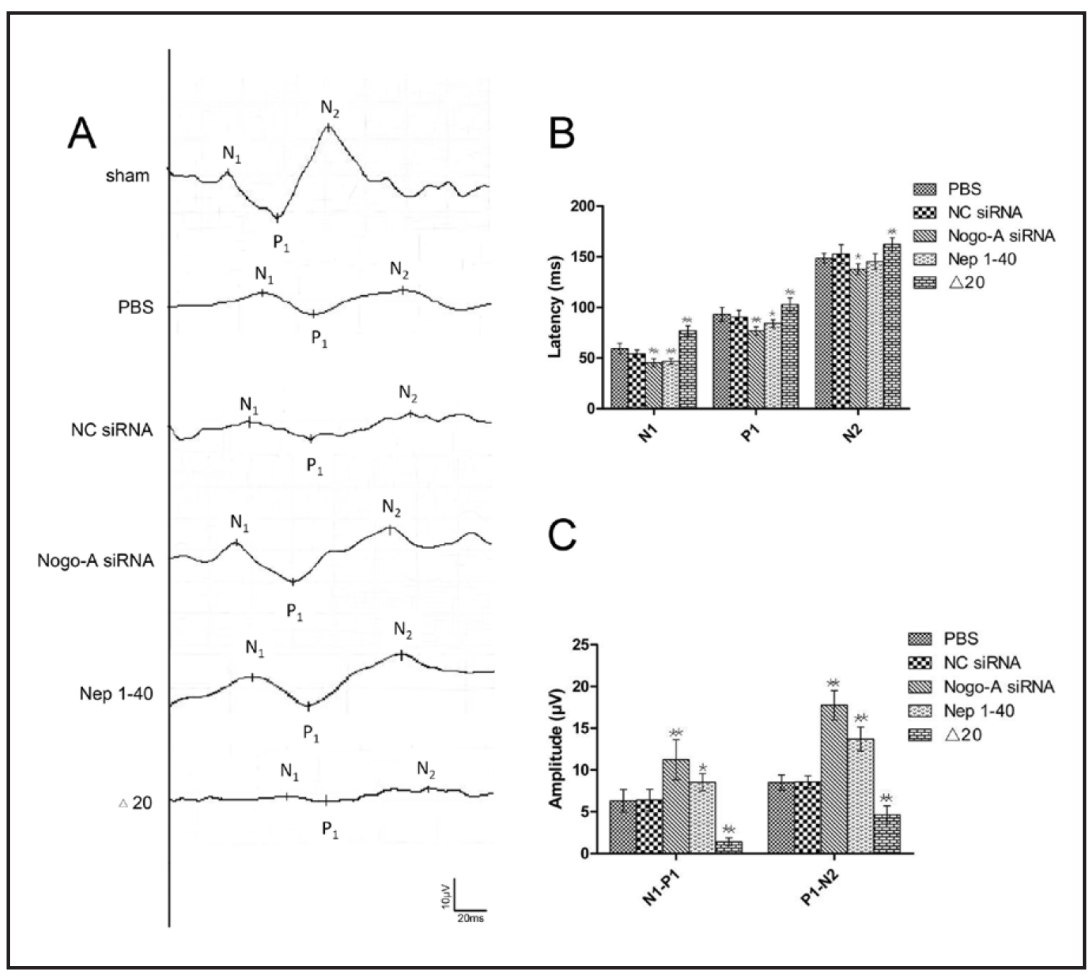

site) were significantly higher in the Nogo-A siRNA ( $p<0.01)$ and Nep1-40 groups $(\mathrm{p}<0.01)$ in comparison to the PBS group, whereas the shortest GAP-43 positive axons were seen in the $\triangle 20$ group compared with the PBS group ( $\mathrm{p}<0.01)$. 
A
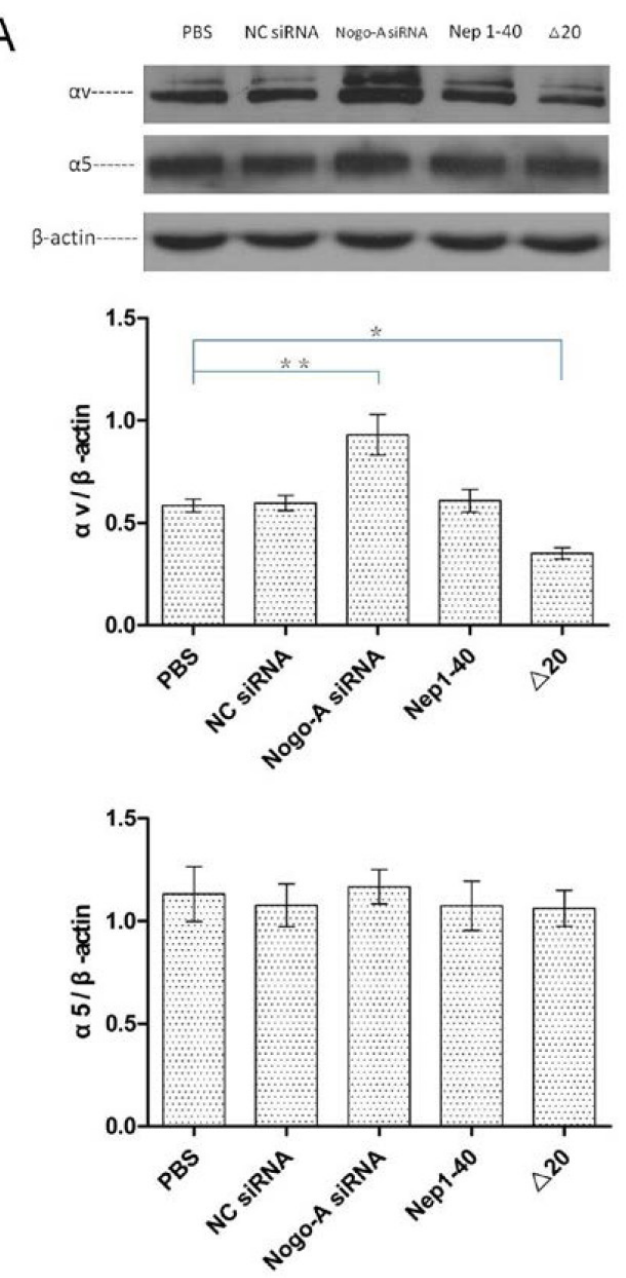
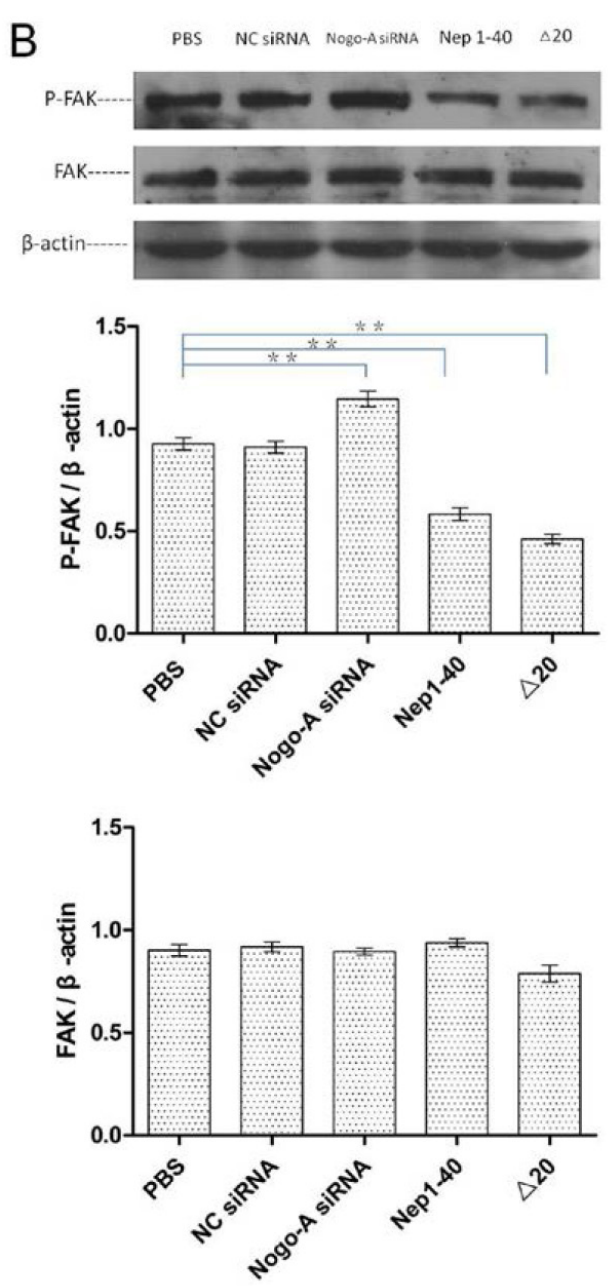

Fig. 5. Representative western blotting and summary data of Amino-Nogo-integrin signaling pathway proteins in the indicated groups. RGCs were treated with NC siRNA, Nogo-A siRNA, Nep1-40 (500 nM) or $\Delta 20$ $(400 \mathrm{nM})$ for 14 days. A: Representative western blotting and summary data showing integrin $\alpha v$ and integrin $\alpha 5$ protein levels in the indicated groups. B: Representative western blotting and summary data showing P-FAK and total FAK protein levels in the indicated groups. Error bar $=\mathrm{SD}, \mathrm{n}=6$. *v. $\mathrm{PBS}$ control, $\mathrm{p}<0.05$; ** vs. PBS control, $\mathrm{p}<0.01$.

\section{Amino-Nogo inhibits axonal function recovery in optic nerve-injured rats}

To evaluate the functional recovery of the injured-optic nerves, we observed the classical NPN wave pattern in the indicated groups (Fig. 4A). We detected the N1, P1 and N2 waves 28 days post-injury (Fig. 4B). Compared with the PBS group, the $\Delta 20$ group showed a significantly prolonged peak latencies of the N1, P1, and N2 waves, but shorter $\mathrm{N} 1$ and P1 waves were observed in the Nogo-A siRNA and Nep1-40 groups. The latency of the N2 wave was shorter in the Nogo-A siRNA group than in the PBS control, whereas the Nep140 group was not significantly different from the control. No significant differences were noted between the PBS and NC siRNA groups. The amplitudes of N1-P1 and P1-N2 (Fig. 4C) decreased in the $\triangle 20$ group compared with the PBS group but recovered in the Nogo-A siRNA and Nep1-40 groups. There was no significant difference in the NC siRNA group in comparison with the PBS group. 


\section{Amino-Nogo attenuates the integrin av signaling pathway}

To determine the underlying signaling pathway, we used western blotting to detect Nogo-A-integrin related proteins (Fig. 5). As a downstream molecule, the phosphorylation of FAK at Tyr397 was also evaluated. Nogo-A siRNA treatment significantly increased integrin $\alpha v$ expression $(p<0.01)$ and the $p$-FAK level $(p<0.01)$ compared with the PBS group. Integrin $\alpha v$ expression was unchanged in the Nepl-40 treatment group, but p-FAK was suppressed $(\mathrm{p}<0.01) . \Delta 20$ treatment significantly attenuated integrin $\alpha v$ expression ( $p$ $<0.05)$ and $\mathrm{p}$-FAK levels $(\mathrm{p}<0.01)$. No significant change in integrin $\alpha 5$ expression or total FAK level were observed in any treatment group.

\section{Discussion}

The optic nerve as a part of CNS, damage to which is difficult to regenerate [20-23]. RGCs axons form the optic nerve, injury of which is one of the causes of visual loss. The pathological basis of visual loss is based on the death of RGCs and the loss of nerve fibers [24, 25]. Axonal degeneration following optic nerve injury causes the death of a large number of RGCs [26], which ultimately leads to irreversible loss of visual function [27]. Therefore, promoting the survival of injured RGCs is the premise and the key to the treatment of optic nerve injury.

Nogo-A is a major myelin-associated inhibitor [2-4] that plays an essential role in axonal regeneration. Our study revealed that Nogo-A siRNA protected against RGC death (Fig. 2). Apart from the survival rate of RGCs, axons regeneration also plays a critical role in vision recovery following optic nerve injury. GAP-43 is a marker of neuronal development that is expressed in the growth cones of regenerating axons [28, 29]. After optic nerve injury, the induction of self-repair can increase immuno-histochemical staining for GAP-43 in regenerating axons [30]. In our study, the number of regenerating axons increased in the Nogo-A siRNA transfected group (Fig. 3). F-VEP was also examined in this study, as it can objectively reflect the degree of optic nerve injury and nerve conduction. The latency of F-VEP reflects the function of nerve conduction and axon myelin sheath integrity, and the amplitude of F-VEP reflects the receptive function of the macula lutea and the number of synaptic contacts between intact axons and their targets [31]. Post-injury, the peak latenies of the N1, P1 and N2 waves increased and the amplitude of N1-P1 and P1-N2 decreased; however, these changes were recovered in the Nogo-A siRNA group (Fig. 4). All of these findings suggest that Nogo-A decreases RGC survival, inhibits axonal regeneration and does harm to the recovery of neurological functions. However, the function of Amino-Nogo may have been characterized more precisely if a specific peptide targeting Amino-Nogo had been used, but such peptide was not yet commercially available.

Nogo-A has two functional domains: the Amino-Nogo domain and the Nogo-66 loop [2, 4-5]. The Nogo-66 domain both inhibits axonal regeneration and regulates axonal growth, guidance and CNS plasticity neurogenesis. Therefore, knocking down Nogo-A expression may affect nerve growth. Therefore, we used Nep1-40 to antagonize the Nogo-66 domain and a recombinant Amino-Nogo functional fragment $(\Delta 20)$ to demonstrate the role of Amino-Nogo in the optic nerve. In our study, Nep1-40 protected against RGC death and $\Delta 20$ reduced the RGC survival rate (Fig. 2). The number of regenerating axons increased in the Nep1-40 injected group, and decreased in the Amino-Nogo injected group (Fig. 3). In the Nep1-40 treatment group, the latencies of the N1and P1 waves were shorter compared with the PBS control, but the N2 latency failed to recover (Fig. 4). This observation indicates that Nogo-66 inhibition is not optimally effective, for promoting regeneration, as endogenous Amino-Nogo still inhibits functional recovery. In addition, obvious changes were detected in the Amino-Nogo injected group, including a minimally visible waveform becoming low and wide (Fig. 4). Taken together, these findings suggest that Amino-Nogo is harmful axonal repair and/or regeneration in injured RGCs, thereby inhibiting the recovery of optic nerve function. 
The most important finding of our study is the elucidation of the underlying mechanism of Amino-Nogo inhibition of the optic nerve following injury. Integrins bind ligands in the extracellular matrix and form adhesion complexes that couple to the actin cytoskeleton. These complexes are necessary for cellular expansion and axonal growth [32]. Amino-Nogo inhibits cell adhesion and axonal outgrowth by inhibiting integrins. Integrins contain several subunits, and it is possible that the Amino-Nogo domain exerts an inhibitory function via a specific integrin subunit $[33,34]$. However, it is unclear how the Amino-Nogo-integrin signaling pathway function in optic nerve regeneration.

Changes in integrin expression can activate downstream molecules [35, 36], such as FAK [37-42]. Our previous study $[12,13]$ showed that Amino-Nogo-integrin signaling pathwayrelated proteins (Nogo-A, integrin $\alpha \mathrm{V}$, integrin $\alpha 5$ and FAK) are widely expressed in the visual system. Results from this study (Fig. 5) clearly showed that Nogo-A siRNA treatment significantly increased integrin $\alpha v$ expression and the p-FAK level, but no changes in total FAK level were observed. In addition, the lack of a significant change in integrin $\alpha 5$ levels in our study may have been because the integrin signaling pathway is regulated by Nogo-A and/or because the integrin subunit involved might be integrin $\alpha \mathrm{v}$ and not integrin $\alpha 5$.

To further clarify the specific function of Nogo-66 and the Amino-Nogo domain, we used Nepl-40 and $\Delta 20$. Western blotting (Fig. 5) revealed that Nepl-40 treatment did not alter integrin $\alpha \mathrm{v}$ expression, but $\Delta 20$ treatment significantly attenuated both integrin $\alpha \mathrm{v}$ expression and the p-FAK level. These findings demonstrate that Amino-Nogo inhibits optic nerve regeneration via an inhibitory effect on the downstream signaling pathway of AminoNogo-integrin $\alpha v$. Moreover, as RhoA regulates FAK activation in turn [43-45], the p-FAK level was decreased in the Nepl-40 treatment group. Together, these results demonstrate that Amino-Nogo inhibits optic nerve regeneration via the integrin $\alpha v$ signaling pathway.

In conclusion, our data suggest that the Amino-Nogo domain decreases RGC survival, inhibits axonal regeneration and inhibits neurological functional recovery, all of which are mediated by the integrin $\alpha v$ signaling pathway. Nonetheless, the results from this study indicate that the Amino-Nogo-integrin signaling pathway is a new molecular mechanism for the promotion of axonal regeneration in the optic nerve, providing new insight into the recovery of visual function after optic nerve injury.

\section{Acknowledgements}

This study was supported by the National Nature Science Foundation of China (No. 31070968 and 81000378). The authors declare that they have no conflict of interest.

\section{Reference}

1 Dowling JE: An approachable part of the brain. Cambridge. The Retina 1987.

$\checkmark 2$ Chen, Huber AB, van der Haar ME, Frank M, Schnell L, Spillmann AA, Christ F, Schwab ME: Nogo-A is a myelin-associated neurite outgrowth inhibitor and an antigen for monoclonal antibody IN-1. Nature 2000;403:434-439.

-3 GrandPre T, Nakamura F, Vartanian T, Strittmatter SM: Identification of the Nogo inhibitor of axon regeneration as a Reticulon protein. Nature 2000;403:439-444.

4 Prinjha R, Moore SE, Vinson M, Blake S, Morrow R, Christie G, Michalovich D, Simmons DL, Walsh FS: Inhibitor of neurite outgrowth in humans. Nature 2000;403:383-384.

-5 Fournier AE, GrandPre T, Strittmatter SM: Identification of a receptor mediating Nogo-66 inhibition of axonal regeneration. Nature 2001;409:341-346.

6 McGee AW, Yang Y, Fischer QS, Daw NW, Strittmatter SM: Experience-driven plasticity of visual cortex limited by myelin and Nogo receptor. Science 2005;309:2222-2226. 
Huo et al.: Amino-Nogo Inhibits Optic Nerve Regeneration

7 Hasegawa T, Ohno K, Sano M, Omura T, Omura K, Nagano A, Sato K: The differential expression patterns of messenger RNAs encoding Nogo-A and Nogo-receptor in the rat central nervous system. Brain Res Mol Brain Res 2005;133:119-130.

8 Klinger M, Taylor JS, Oertle T, Schwab ME, Stuermer CA, Diekmann H: Identification of Nogo-66 receptor (NgR) and homologous genes in fish. Mol Biol Evol 2004;21:76-85.

9 O'Neill P, Whalley K, Ferretti P: Nogo and Nogo-66 receptor in human and chick: implications for development and regeneration. Dev Dyn 2004;231:109-121.

$\checkmark 10$ Al Halabiah H, Delezoide AL, Cardona A, Moalic JM, Simonneau M: Expression pattern of NOGO and NgR genes during human development. Gene Expr Patterns 2005;5:561-568.

11 Mingorance A, Fontana X, Sole M, Burgaya F, Urena JM, Teng FY, Tang BL, Hunt D, Anderson PN, Bethea JR, Schwab ME, Soriano E, del Rio JA: Regulation of Nogo and Nogo receptor during the development of the entorhino-hippocampal pathway and after adult hippocampal lesions. Mol Cell Neurosci 2004;26:34-49.

12 Huo Y, Yin XL, Ji SX, Zou H, Lang M, Zheng Z, Cai XF, Liu W, Chen CL, Zhou YG, Yuan RD, Ye J: Inhibition of retinal ganglion cell axonal outgrowth through the Amino-Nogo-A signaling pathway. Neurochem Res 2013;38:1365-1374.

13 Yin XL, Yuan RD, Ji SX, Ye J: The expression patterns of Nogo-A and NgR in the neonatal rat visual nervous system. Neuroch Res 2009;34:1204-1208.

14 Yoles E, Schwartz M: Degeneration of spared axons following partial white matter lesion: implications for optic nerve neuropathies. Exp Neurol 1998;153:1-7.

15 Cho KS, Yang L, Lu B, Feng Ma H, Huang X, Pekny M, Chen DF: Re-establishing the regenerative potential of central nervous system axons in postnatal mice. J Cell Sci 2005;118:863-872.

-16 Karim MZ, Sawada A, Mizuno K, Kawakami H, Ishida K, Yamamoto T: Neuroprotective effect of nipradilol [3,4-dihydro-8-(2-hydroxy-3-isopropylamino)-propoxy-3-nitroxy-2H-1-benzopyran] in a rat model of optic nerve degeneration. J Glaucoma 2009;18:26-31.

17 Chen C, Chen XF, Yin XL, Yuan RD, Wang BC, Ye J: NgR RNA interference, combined with zymosan intravitreal injection, enhances optic nerve regeneration. J Neurochem 2009;110:1628-1634.

18 Planchamp V, Bermel C, Tonges L, Ostendorf T, Kugler S, Reed JC, Kermer P, Bahr M, Lingor P: BAG1 promotes axonal outgrowth and regeneration in vivo via Raf- 1 and reduction of ROCK activity. Brain 2008;131:2606-2619.

19 Schaden H, Stuermer CA, Bahr M: GAP-43 immunoreactivity and axon regeneration in retinal ganglion cells of the rat. J Neurobiol 1994;25:1570-1578.

20 Snow DM, Lemmon V, Carrino DA, Caplan AI, Silver J: Sulfated proteoglycans in astroglial barriers inhibit neurite outgrowth in vitro. Exp Neurol 1990;109:111-130.

-21 Schnell L, Schneider R, Kolbeck R, Barde YA, Schwab ME: Neurotrophin-3 enhances sprouting of corticospinal tract during development and after adult spinal cord lesion. Nature 1994;367:170-173.

22 Tuszynski MH, Gage FH: Maintaining the neuronal phenotype after injury in the adult CNS. Neurotrophic factors, axonal growth substrates, and gene therapy. Mol Neurobiol 1995;10:151-167.

23 Fawcett J: Molecular control of brain plasticity and repair. Prog Brain Res 2009;175:501-509.

24 Harvey AR, Hu Y, Leaver SG, Mellough CB, Park K, Verhaagen J, Plant GW, Cui Q: Gene therapy and transplantation in CNS repair: the visual system. Prog Ret Eye Res 2006;25:449-489.

25 Dahlmann-Noor AH, Vijay S, Limb GA, Khaw PT: Strategies for optic nerve rescue and regeneration in glaucoma and other optic neuropathies. Drug Discov Today 2010;15:287-299.

26 Kermer P, Klocker N, Weishaupt JH, Bahr M: Transection of the optic nerve in rats: studying neuronal death and survival in vivo. Brain Research. Brain Res Protocols 2001;7:255-260.

27 Gellrich NC, Schimming R, Zerfowski M, Eysel UT: Quantification of histological changes after calibrated crush of the intraorbital optic nerve in rats. Brit J Ophthalmol 2002;86:233-237.

28 Perrone-Bizzozero NI, Sower AC, Bird ED, Benowitz LI, Ivins KJ, Neve RL: Levels of the growth-associated protein GAP-43 are selectively increased in association cortices in schizophrenia. Proc Nat Acad Sci USA 1996;93:14182-14187.

29 Dusart I, Ghoumari A, Wehrle R, Morel MP, Bouslama-Oueghlani L, Camand E, Sotelo C: Cell death and axon regeneration of Purkinje cells after axotomy: challenges of classical hypotheses of axon regeneration. Brain Research. Brain Res Rev 2005;49:300-316.

-30 Plunet W, Kwon BK, Tetzlaff W: Promoting axonal regeneration in the central nervous system by enhancing the cell body response to axotomy. J Neurosc Res 2002;68:1-6. 
Huo et al.: Amino-Nogo Inhibits Optic Nerve Regeneration

-31 Maier J, Dagnelie G, Spekreijse H, van Dijk BW: Principal components analysis for source localization of VEPs in man. Vision Res 1987;27:165-177.

- 32 Beningo KA, Dembo M, Kaverina I, Small JV, Wang YL: Nascent focal adhesions are responsible for the generation of strong propulsive forces in migrating fibroblasts. J Cell Biol 2001; 153: 881-888.

-33 Hu F, Strittmatter SM: The N-terminal domain of Nogo-A inhibits cell adhesion and axonal outgrowth by an integrin-specific mechanism. J Neurosci 2008;28:1262-1269.

34 Tan CL, Kwok JC, Patani R, Ffrench-Constant C, Chandran S, Fawcett JW: Integrin activation promotes axon growth on inhibitory chondroitin sulfate proteoglycans by enhancing integrin signaling. J Neurosci 2011;31:6289-6295.

35 Chen Q, Meng LH, Zhu CH, Lin LP, Lu H, Ding J: ADAM15 suppresses cell motility by driving integrin alpha5beta1 cell surface expression via Erk inactivation. Int J Biochem Cell Biol 2008;40:2164-2173.

-36 Shen X, Falzon M: PTH-related protein upregulates integrin alpha6beta4 expression and activates Akt in breast cancer cells. Exp Cell Res 2006;312:3822-3834.

37 Guan JL: Role of focal adhesion kinase in integrin signaling. Int J Biochem Cell Biol 1997;29:1085-1096.

- 38 Ilic D, Damsky CH, Yamamoto T: Focal adhesion kinase: at the crossroads of signal transduction. J Cell Sci 1997;110:401-407.

-39 McLean GW, Fincham VJ, Frame MC: v-Src induces tyrosine phosphorylation of focal adhesion kinase independently of tyrosine 397 and formation of a complex with Src. J Biol Chem 2000;275:23333-23339.

40 Rico B, Beggs HE, Schahin-Reed D, Kimes N, Schmidt A, Reichardt LF: Control of axonal branching and synapse formation by focal adhesion kinase. Nat Neurosci 2004;7:1059-1069.

-41 Robles E, Gomez TM: Focal adhesion kinase signaling at sites of integrin-mediated adhesion controls axon pathfinding. Nat Neurosci 2006;9:1274-1283.

42 Gonzenbach RR, Schwab ME: Disinhibition of neurite growth to repair the injured adult CNS: focusing on Nogo. Cell Mol Life Sci 2008;65:161-176.

43 Kumagai N, Morii N, Fujisawa K, Nemoto Y, Narumiya S: ADP-ribosylation of rho p21 inhibits lysophosphatidic acid-induced protein tyrosine phosphorylation and phosphatidylinositol 3-kinase activation in cultured Swiss 3T3 cells. J Biol Chem 1993;268:24535-24538.

-44 Barry ST, Flinn HM, Humphries MJ, Critchley DR, Ridley AJ: Requirement for Rho in integrin signalling. Cell Adhes Commun 1997;4:387-398.

45 Torsoni AS, Marin TM, Velloso LA, Franchini KG: RhoA/ROCK signaling is critical to FAK activation by cyclic stretch in cardiac myocytes. Am J Physiol Heart Circ 2005;289:1488-1496. 